\title{
Solvability of nonlinear fractional integro-differential equation with nonlocal condition
}

Solvability of nonlinear fractional equation

\author{
Sakhri Aicha and Ahcene Merad \\ Department of Mathematics and Computer Sciences, Larbi Ben Mhidi University, \\ Oum El Bouaghi, Algeria
}

\begin{abstract}
Purpose - This study describes the applicability of the $a$ priori estimate method on a nonlocal nonlinear fractional differential equation for which the weak solution's existence and uniqueness are proved. The authors divide the proof into two sections for the linear associated problem; the authors derive the a priori bound and demonstrate the operator range density that is generated. The authors solve the nonlinear problem by introducing an iterative process depending on the preceding results.

Design/methodology/approach - The functional analysis method is the a priori estimate method or energy inequality method.

Findings - The results show the efficiency of a priori estimate method in the case of time-fractional order differential equations with nonlocal conditions. Our results also illustrate the existence and uniqueness of the continuous dependence of solutions on fractional order differential equations with nonlocal conditions.

Research limitations/implications - The authors' work can be considered a contribution to the development of the functional analysis method that is used to prove well-positioned problems with fractional order.

Originality/value - The authors confirm that this work is original and has not been published elsewhere, nor is it currently under consideration for publication elsewhere.
\end{abstract}

Keywords Existence and uniqueness, A priori estimate, Fractional derivatives and integrals, Integral condition

Paper type Research paper

\section{Introduction}

Fractional order partial differential equations have become one of the most popular areas of research in mathematical analysis. Their application has been utilized in various scientific fields, such as optimal control theory, chemistry, physics, mathematics, biology, finance and engineering [1-5].

Integro-differential equations are a combination of derivatives and integrals which are appealing to both researchers and scientists for their applications in many areas [6-9]. Numerous mathematical formulations of physical phenomena include integro-differential equations, which may arise in modelling biological fluid dynamics [10-15].

It is important to establish effective methods to solve fractional differential equations (FDEs). Recently, a great deal of attention was dedicated to FDE solutions utilizing different methods, including the Adomian decomposition method [16,17], the Laplace transform

\section{JEL Classification - 34A12, 35B45}

(C) Sakhri Aicha and Ahcene Merad. Published in Arab Journal of Mathematical Sciences. Published by Emerald Publishing Limited. This article is published under the Creative Commons Attribution (CC BY 4.0) licence. Anyone may reproduce, distribute, translate and create derivative works of this article (for both commercial and non-commercial purposes), subject to full attribution to the original publication and authors. The full terms of this licence may be seen at http://creativecommons.org/licences/by/4.0/ legalcode
Received 22 May 2021 Revised 28 August 2021 Accepted 9 September 2021

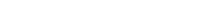


method [18], exponential differential operators [19], the F-expansion method [20], non-Nehari manifold method [21] and the reproducing kernel space method [22,23], in the search for exact or analytical solutions. The applicability of most techniques becomes difficult with the presence of the integral condition. The energy inequality method is a useful tool for studying nonlocal fractional and classical problems. Compared with other techniques, it has an essential role in establishing the solution's existence and uniqueness proof and depends on density arguments and certain a priori bounds.

There have been few articles related to nonlinear fractional partial equations that employ the energy inequality method [24]. Furthermore, for partial differential equations with classical order, many results have utilized this method [25-28]. Motivated by the previous results, the authors studied a nonlocal nonlinear time-fractional order problem. Moreover, we demonstrate the solution's uniqueness, existence and dependence on the given data.

This article is outlined in the following way: in Section 2, we present the main problem. The next section is focused on posing the associated linear problem and introducing some required preliminaries and functional spaces. Then, in Section 4, we develop the energy inequality method to demonstrate the linear problem's strong solution's uniqueness. In addition, we prove the strong solution's existence in Section 5. Moreover, we derive a priori bound and demonstrate the generated operator range density in a Hilbert space. We solve the nonlinear problem in Section 6 by utilizing the results achieved in Sections 4 and 5, and an iteration process.

\section{Statement of problem}

In the region $D=\Omega \times[0, T], \Omega=(0,1), T<\infty$, we pose the nonlinear fractional equation

$$
\begin{aligned}
\mathcal{L} v & ={ }^{C} \partial_{t}^{\beta+1} v-\frac{\partial}{\partial x}\left(\gamma(x, t) \frac{\partial v}{\partial x}\right)-\frac{\partial^{2}}{\partial x \partial t}\left(\eta(x, t) \frac{\partial v}{\partial x}\right)-\int_{0}^{t} \xi(t-z) v(x, z) d z \\
& =f\left(x, t, v, \frac{\partial v}{\partial x}\right)
\end{aligned}
$$

with $0<\beta<1$.

Associated with initial condition

$$
\ell_{1} v=v(x, 0)=\varphi(x), \quad \ell_{2} v=\frac{\partial v(x, 0)}{\partial t}=\psi(x), \quad x \in \Omega,
$$

and the boundary condition

$$
\int_{0}^{1} v(x, t) d x=0, \quad v_{x}(1, t)=0, \quad t \in(0, T),
$$

Such that the known functions $\gamma, \eta$ and $\xi$ verify Assumption 1, and data functions $f, \varphi$ and $\psi$ belong to suitable function spaces as mentioned in Section 3.

In the Caputo definition for a function $v$, the fractional derivatives of order $\beta+1$ with $0<\beta<1$ is defined as

where $\Gamma($.$) is the gamma function$

$$
{ }^{C} \partial_{t}^{\beta+1} v(x, t)=\frac{1}{\Gamma(1-\beta)} \int_{0}^{1} \frac{v_{\tau \tau}(x, \tau)}{(t-\beta)^{\beta}} d \tau,
$$


and the Riemann-Liouville integral of order $0<\beta<1$, which is given by

$$
D_{t}^{-\beta} v(x, t)=\frac{1}{\Gamma(\beta)} \int_{0}^{1} \frac{v(x, \tau)}{(t-\beta)^{1-\beta}} d \tau \text {. }
$$

Solvability of nonlinear fractional equation

\section{Technical tools and associated linear problem}

We define some function spaces and tools required to investigate the following linear problem associated with problems (1)-(3).

$$
\begin{gathered}
\mathcal{L} v={ }^{C} \partial_{t}^{\beta+1} v-\frac{\partial}{\partial x}\left(\gamma(x, t) \frac{\partial v}{\partial x}\right)-\frac{\partial^{2}}{\partial x \partial t}\left(\eta(x, t) \frac{\partial v}{\partial x}\right)-\int_{0}^{t} \xi(t-z) v(x, z) d z=f(x, t) \\
\ell_{1} v=v(x, 0)=\varphi(x), \quad \ell_{2} v=\frac{\partial v(x, 0)}{\partial t}=\psi(x), \quad x \in \Omega, \\
\int_{0}^{1} v(x, t) d x=0, \quad v_{x}(1, t)=0, \quad t \in(0, T),
\end{gathered}
$$

First, we convert problems (1)-(3) into an equivalent operator form

$$
L v=\mathcal{F}=(f, \varphi, \psi)
$$

where the unbounded operator $L=\left(\mathcal{L}, \ell_{1}, \ell_{2}\right)$ with $L: E \rightarrow F$ is defined in $D(L)$ such that

$$
D(L)=\left\{\begin{array}{l}
v \in L^{2}(D),{ }^{c} \partial_{t}^{\beta+1} v, \partial v / \partial t, \partial v / \partial x, \partial^{2} v / \partial x^{2}, \partial^{3} v / \partial x^{2} \partial t \in L^{2}(D) \\
\int_{0}^{1} v(x, t) d x=0, \quad v_{x}(1, t)=0, t \in(0, T),
\end{array}\right.
$$

and $v$ also verify the initial condition. Here $E$ is Banach space containing elements having the finite norm

$$
\|v\|_{E}^{2}=D_{t}^{\beta-1}\left\|\Im_{x} \frac{\partial v}{\partial t}\right\|_{L^{2}(\Omega)}^{2}+\left\|\frac{\partial v}{\partial t}\right\|_{L^{2}(D)}^{2}+\|v\|_{C\left((0, T), L^{2}(\Omega)\right)}^{2}
$$

and $F$ is Hilbert space composed of functions normed with

$$
\|\mathcal{F}\|_{F}^{2}=\left\|\Im_{x} f\right\|_{L^{2}(D)}^{2}+\|\varphi\|_{L^{2}(\Omega)}^{2}+\left\|\Im_{x} \psi\right\|_{L^{2}(\Omega)}^{2} .
$$

Lemma 1. [29] Let $S(t)$ a nonnegative absolute continuous function verifying the inequality

$$
{ }^{C} \partial_{t}^{\alpha} S(t) \leq c_{1} S(t)+c_{2}(t), \quad 0<\alpha<1,
$$

for almost all $t \in[0, T]$, where $c_{1}$ is a positive constant and $c_{2}(t)$ is an integrable nonnegative function on $[0, T]$. Then,

$$
S(t) \leq S(0) E_{\alpha}\left(c_{1} t^{\alpha}\right)+\Gamma(\alpha) E_{\alpha, \alpha}\left(c_{1} t^{\alpha}\right) D_{t}^{-\alpha} c_{2}(t),
$$

where

are Mittag-Leffler functions.

$$
E_{\alpha}(x)=\sum_{n=0}^{\infty} \frac{x^{n}}{\Gamma(\alpha n+1)} \quad \text { and } \quad E_{\alpha, \nu}(x)=\sum_{n=0}^{\infty} \frac{x^{n}}{\Gamma(\alpha n+\nu)},
$$


Lemma 2. [29] On the interval [0, T], any absolute continuous function $y(t)$ verifies the following estimate:

$$
S(t)^{C} \partial_{t}^{\beta} S(t) \geq \frac{1}{2} c \partial_{t}^{\beta} S^{2}(t), \quad 0<\beta<1,
$$

Lemma 3. [30] For any $n \in \mathbb{N}$, we have

$$
\left\|\mathfrak{\Im}_{x}^{2 n} v\right\|_{L^{2}(0, l)}^{2} \leq\left(\frac{l}{2}\right)^{2 n}\|v\|_{L^{2}(0, l)}^{2}
$$

where

$$
\Im_{x}^{2 n} v=\int_{0}^{x} \int_{0}^{\xi_{1}} \ldots \int_{0}^{\xi_{2 n-1}} v(\eta, t) d \eta d \xi_{2 n-1} \ldots d \xi_{1}=\int_{0}^{x} \frac{(x-\xi)^{2 n-1}}{(2 n-1) !} v(\xi, t) d \xi .
$$

Cauchy $\varepsilon$-inequality [31]

$$
|a b| \cdot \leq \frac{\varepsilon}{2} a^{2}+\frac{1}{2 \varepsilon} b^{2}
$$

which holds for arbitrary $a$ and $b$, and all $\varepsilon>0$.

\section{A priori estimate and consequences}

Assumption 1. For any $(x, t) \in D$, we suppose that

$$
\begin{gathered}
c_{0} \leq \gamma(x, t) \leq c_{1}, \frac{\partial \gamma(x, t)}{\partial t} \leq c_{2}, \frac{\partial \gamma(x, t)}{\partial x} \leq c_{3} \\
c_{4} \leq \eta(x, t), c_{5} \leq \frac{\partial \eta(x, t)}{\partial t} \leq c_{6}, \frac{\partial \eta(x, t)}{\partial x} \leq c_{7} \\
\frac{\partial^{2} \eta(x, t)}{\partial t^{2}} \leq c_{8}, \frac{\partial^{2} \eta(x, t)}{\partial t \partial x} \leq c_{9}, \xi(x, t) \leq c_{10}
\end{gathered}
$$

such that $c_{i}(i=0, \ldots, 10)$ are positive constants.

Theorem 4. Let Assumption 1 be fulfilled. Then, any function $v \in D(L)$ verify the following estimate

$$
\|v\|_{E} \leq C\|L v\|_{F}
$$

where $C>0$ constant independent of $v$.

Proof. We take the scalar product $L^{2}\left(D^{\tau}\right)$ of equality (6) and the integro-differential operator $M v=-2 \mathfrak{\Im}_{x}^{2} \frac{\partial v}{\partial t}$, such that $\tau \in[0, T]$, we have 


$$
\begin{aligned}
& 2\left({ }^{C} \partial_{t}^{\beta+1} v,-\Im_{x}^{2} \frac{\partial v}{\partial t}\right)_{L^{2}\left(D^{\tau}\right)}-2\left(\frac{\partial}{\partial x}\left(\gamma(x, t) \frac{\partial v}{\partial x}\right),-\Im_{x}^{2} \frac{\partial v}{\partial t}\right)_{L^{2}\left(D^{\tau}\right)} \\
& -2\left(\frac{\partial^{2}}{\partial x \partial t}\left(\eta(x, t) \frac{\partial v}{\partial x}\right),-\Im_{x}^{2} \frac{\partial v}{\partial t}\right)_{L^{2}\left(D^{\tau}\right)}-2\left(\int_{0}^{t} \xi(t-z) v(x, z) d z,-\Im_{x}^{2} \frac{\partial v}{\partial t}\right)_{L^{2}\left(D^{\tau}\right)} \\
& =2\left(f(x, t),-\Im_{x}^{2} \frac{\partial v}{\partial t}\right)_{L^{2}\left(D^{\tau}\right)} .
\end{aligned}
$$

The integration of the first three terms on the left-hand side (LHS) of Equation (20), taking into consideration initial and boundary conditions (2) - (3), gives

$$
\begin{aligned}
& -\left({ }^{c} \partial_{t}^{\beta+1} v,-\Im_{x}^{2} \frac{\partial v}{\partial t}\right)_{L^{2}\left(D^{\tau}\right)}=\left({ }^{c} \partial_{t}^{\beta} \frac{\partial v}{\partial t}, \Im^{2} \frac{\partial v}{\partial t}\right)_{L^{2}\left(D^{\tau}\right)}=2\left({ }^{c} \partial_{t}^{\beta}\left(\Im \frac{\partial v}{\partial t}\right), \Im_{x} \frac{\partial v}{\partial t}\right)_{L^{2}\left(D^{\tau}\right)} \\
& -2\left(\frac{\partial}{\partial x}\left(\gamma(x, t) \frac{\partial v}{\partial x}\right),-\mathfrak{\Im}_{x}^{2} v_{t}\right)_{L^{2}\left(D^{\tau}\right)}=-\int_{D^{\tau}} \frac{\partial \gamma}{\partial t} v^{2} d x d t+2 \int_{D^{\tau}} \frac{\partial \gamma}{\partial x} v \mathfrak{\Im}_{x} \frac{\partial v}{\partial t} d x d t \\
& +\int_{0}^{1} \gamma(x, \tau) v^{2}(x, \tau) d x-\int_{0}^{1} \gamma(x, 0) \varphi^{2}(x) d x \\
& -2\left(\frac{\partial^{2}}{\partial x \partial t}\left(\eta(x, t) \frac{\partial v}{\partial x}\right),-\Im_{x}^{2} \frac{\partial v}{\partial t}\right)_{L^{2}\left(D^{\tau}\right)}=2 \int_{D^{\tau}} \eta\left(\frac{\partial v}{\partial t}\right)^{2} d x d t+\int_{0}^{1} \frac{\partial \eta(x, \tau)}{\partial t} v^{2}(x, \tau) d x \\
& -\int_{D^{\tau}} \frac{\partial^{2} \eta}{\partial t^{2}} v^{2} d x d t+2 \int_{D^{\tau}} \frac{\partial \eta}{\partial x} \frac{\partial v}{\partial t} \Im_{x} \frac{\partial v}{\partial t} d x d t+2 \int_{D^{\tau}} \frac{\partial^{2} \eta}{\partial x \partial t} v \Im_{\Im_{x}} \frac{\partial v}{\partial t} d x d t-\int_{0}^{1} \frac{\partial \eta(x, 0)}{\partial t} \varphi^{2}(x) d x
\end{aligned}
$$

Substituting (21)-(23) into (20) yields

$$
\begin{aligned}
& 2\left({ }^{C} \partial_{t}^{\beta}\left(\Im_{\Im_{x}} \frac{\partial v}{\partial t}\right), \Im_{x} \frac{\partial v}{\partial t}\right)_{L^{2}\left(D^{\tau}\right)}+2 \int_{D^{\tau}} \eta\left(\frac{\partial v}{\partial t}\right)^{2} d x d t+\int_{0}^{1}\left(\gamma(x, \tau)+\frac{\partial \eta(x, \tau)}{\partial t}\right) v^{2}(x, \tau) d x= \\
& 2\left(f(x, t),-\Im_{x}^{2} \frac{\partial v}{\partial t}\right)_{L^{2}\left(D^{\tau}\right)}+2\left(\int_{0}^{t} \xi(t-z) v(x, z) d z,-\Im^{2} \frac{\partial v}{\partial t}\right)_{L^{2}\left(D^{\tau}\right)}+\int_{D^{\tau}}\left(\frac{\partial \gamma}{\partial t}+\frac{\partial^{2} \eta}{\partial t^{2}}\right) v^{2} d x d t \\
& +\int_{0}^{1}\left(\gamma(x, 0)+\frac{\partial \eta(x, 0)}{\partial t}\right) \varphi^{2}(x) d x-2 \int_{D^{\tau}} \frac{\partial \eta}{\partial x} \frac{\partial v}{\partial t} \Im_{x} \frac{\partial v}{\partial t} d x d t-2 \int_{D^{\tau}}\left(\frac{\partial \gamma}{\partial x}+\frac{\partial^{2} \eta}{\partial x \partial t}\right) v \Im_{x} \frac{\partial v}{\partial t} d x d t
\end{aligned}
$$

By applying inequality (16), we estimate the first and the last two terms on the right-hand side (RHS) of (24); as such it follows that

$$
-2 \int_{D^{\tau}} f \Im^{2} \frac{\partial v}{\partial t} d x d t \leq \int_{D^{\tau}}\left(\mathfrak{\Im}_{x} f\right)^{2} d x d t+\int_{D^{\tau}}\left(\Im_{x} \frac{\partial v}{\partial t}\right)^{2} d x d t
$$


AJMS

$$
\begin{aligned}
& 2 \int_{D^{\tau}}\left(\int_{0}^{t} \xi(t-z) v(x, z) d z\right) \mathfrak{\Im}_{x}^{2} \frac{\partial v}{\partial t} d x d t \leq c_{10} T^{2}\|v\|_{L^{\left(D^{\tau}\right)}}^{2}+\frac{1}{2} \int_{D^{\tau}}\left(\Im_{\Im_{x}} \frac{\partial v}{\partial t}\right)^{2} d x d t \\
& -2 \int_{D^{x}}\left(\frac{\partial \gamma}{\partial x}+\frac{\partial^{2} \eta}{\partial x \partial t}\right) v \Im_{x} \frac{\partial v}{\partial t} d x d t \leq 2 \int_{D^{x}}\left\{\left(\frac{\partial \gamma}{\partial x}\right)^{2}+\left(\frac{\partial^{2} \eta}{\partial x \partial t}\right)^{2}\right\} v^{2} d x d t+\int_{D^{x}}\left(\Im_{x} \frac{\partial v}{\partial t}\right)^{2} d x d t, \\
& -2 \int_{D^{x}} \frac{\partial \eta}{\partial x} \frac{\partial v}{\partial t} \Im_{x} \frac{\partial v}{\partial t} d x d t \leq c_{4} \int_{D^{x}}\left(\frac{\partial v}{\partial t}\right)^{2} d x d t+\frac{1}{c_{4}} \int_{D^{x}}\left(\frac{\partial \eta}{\partial x}\right)^{2}\left(\Im_{x} \frac{\partial v}{\partial t}\right)^{2} d x d t .
\end{aligned}
$$

By Lemma 2, the first term on the LHS of (20) becomes

$$
2\left({ }^{c} \partial_{t}^{\beta}\left(\mathfrak{\Im}_{x} \frac{\partial v}{\partial t}\right), \Im_{x} \frac{\partial v}{\partial t}\right)_{L^{2}\left(D^{r}\right)} \geq \int_{D^{x}} \partial_{t}^{\beta}\left(\Im_{x} \frac{\partial v}{\partial t}\right)^{2} d x d t
$$

Hence, by Formulas (25)-(29) and Assumption (1), we obtain

$$
\begin{aligned}
& \int_{D^{x}}\left({ }^{c} \partial_{t}^{\beta}\left(\Im_{\Im} \frac{\partial v}{\partial t}\right)^{2}\right) d x d t+\int_{0}^{\tau}\left\|\frac{\partial v}{\partial t}(., t)\right\|_{L^{2}(\Omega)}^{2} d t+\|v(., \tau)\|_{L^{2}(\Omega)}^{2} \\
\leq & \delta_{1}\left\{\int_{0}^{\tau}\left\|\Im_{x} f(., t)\right\|_{L_{(\Omega)}}^{2} d t+\|\varphi\|_{L^{2}(\Omega)}^{2}+\int_{0}^{\tau}\left\|\Im_{x} \frac{\partial v}{\partial t}(., t)\right\|_{L_{(\Omega)}}^{2} d t\right\} \\
+ & \delta_{2}\left\{\int_{0}^{\tau}\|v(., t)\|_{L^{2}(\Omega)}^{2} d t\right\},
\end{aligned}
$$

where

$$
\begin{gathered}
\delta_{1}=\frac{\max \left(1, c_{1}+c_{6}, \frac{5}{2}+\frac{c_{7}^{2}}{c_{4}}\right)}{\min \left(c_{4}, c_{0}+c_{5}, 1\right)}, \\
\delta_{2}=\frac{c_{2}+c_{8}+c_{3}^{2}+c_{9}^{2}+c_{10} T^{2}}{\min \left(c_{4}, c_{6}+c_{5}, 1\right)} .
\end{gathered}
$$

Now, since

$$
\int_{0}^{\tau} c \partial_{t}^{\beta}\left\|\Im_{x} \frac{\partial v^{2}}{\partial t}\right\|_{L^{2}(\Omega)} d t=D_{\tau}^{\beta-1}\left\|\Im_{x} \frac{\partial v}{\partial t}\right\|_{L^{2}(\Omega)}^{2}-\frac{\tau^{1-\beta}}{(1-\beta) \Gamma(1-\beta)}\left\|\Im_{x} \psi\right\|_{L^{2}(\Omega)}^{2},
$$

then

$$
\begin{aligned}
& D_{\tau}^{\beta-1}\left\|\Im_{x} \frac{\partial v}{\partial t}\right\|_{L^{2} \Omega}^{2}+\int_{0}^{\tau}\left\|\frac{\partial v}{\partial t}(., t)\right\|_{L^{2}(\Omega)}^{2} d t+\|v(., \tau)\|_{L^{2}(\Omega)}^{2} \\
\leq & \delta_{3}\left\{\int_{0}^{\tau}\left\|\Im_{x} f(., t)\right\|_{L_{(\Omega)}}^{2} d t+\|\varphi\|_{L^{2}(\Omega)}^{2}+\left\|\Im_{x} \psi\right\|_{L^{2}(\Omega)}^{2}+\int_{0}^{\tau}\left\|\Im_{x} \frac{\partial v}{\partial t}(., t)\right\|_{L(\Omega)}^{2} d t\right\} \\
+ & \delta_{2}\left\{\int_{0}^{\tau}\|v(., t)\|_{L^{2}(\Omega)}^{2} d t,\right\}
\end{aligned}
$$


where

$$
\delta_{3}=\max \left(\delta_{1}, \frac{T^{1-\beta}}{(1-\beta) \Gamma(1-\beta)}\right)
$$

We need to drop the last term on the RHS of (31). Therefore, we use Gronwall's lemma, which yields

$$
\begin{aligned}
& D_{\tau}^{\beta-1}\left\|\Im_{x} \frac{\partial v}{\partial t}\right\|_{L^{2}(\Omega)}^{2}+\int_{0}^{\tau}\left\|\frac{\partial v}{\partial t}(., t)\right\|_{L^{2}(\Omega)}^{2} d t+\|v(., \tau)\|_{L^{2}(\Omega)}^{2} \\
\leq & \delta_{4}\left\{\int_{0}^{\tau}\left\|\Im_{x} f(., t)\right\|_{L(\Omega)}^{2} d+\|\varphi\|_{L^{2}(\Omega)}^{2} t+\left\|\Im_{x} \psi\right\|_{L^{2}(\Omega)}^{2}+\int_{0}^{\tau}\left\|\Im_{x} \frac{\partial v}{\partial t}(., t)\right\|_{L(\Omega)}^{2} d t\right\},
\end{aligned}
$$

where

$$
\delta_{4}=\exp \left(\delta_{2} T\right) \delta_{3}
$$

Now, by discarding the last two terms on the LHS of (32) then posing $S(\tau)=$ $\int_{0}^{\tau}\left\|\Im_{x} \frac{\partial v}{\partial t}(., t)\right\|_{L^{2}(\Omega)}^{2} d t,{ }^{C} \partial_{t}^{\beta+1} S(\tau)=D_{\tau}^{\beta-1}\left\|\Im_{x} \frac{\partial v}{\partial t}\right\|_{L^{2}(\Omega)}^{2}$, with $S(0)=0$, in Lemma (1), we obtain

$$
\int_{0}^{\tau}\left\|\mathfrak{\Im}_{x} \frac{\partial v}{\partial t}(., t)\right\|_{L^{2}(\Omega)}^{2} d t \leq \delta_{5}\left\{D_{\tau}^{-1-\beta}\left\|\mathfrak{\Im}_{x} f\right\|_{L(\Omega)}^{2}+\|\varphi\|_{L^{2}(\Omega)}^{2}+\left\|\mathfrak{\Im}_{x} \psi\right\|_{L^{2}(\Omega)}^{2}\right\},
$$

where

$$
\delta_{5}=\Gamma(\beta) E_{\beta, \beta}\left(c_{17} T^{\beta}\right) \max \left(1, \frac{T^{(\beta+1)}}{(1+\beta) \Gamma(1+\beta)}\right)
$$

Combining (32)-(33) yields

$$
\begin{aligned}
& D_{\tau}^{\beta-1}\left\|\Im_{x} \frac{\partial v}{\partial t}\right\|_{L^{2}(\Omega)}^{2}+\int_{0}^{\tau}\left\|\frac{\partial v}{\partial t}(., t)\right\|_{L^{2}(\Omega)}^{2} d t+\|v(., \tau)\|_{L^{2}(\Omega)}^{2} \\
\leq & \delta_{6}\left\{D_{\tau}^{-\beta-1}\left\|\Im_{x} f\right\|_{L^{2}(\Omega)}^{2}+\int_{0}^{\tau}\left\|\Im_{x} f(., t)\right\|_{L(\Omega)}^{2} d t+\|\varphi\|_{L^{2}(\Omega)}^{2}+\left\|\Im_{x} \psi\right\|_{L^{2}(\Omega)}^{2}\right\},
\end{aligned}
$$

where

$$
\delta_{6}=\max \left(\delta_{4} \delta_{5}, \delta_{5}\right)
$$

From given inequality

$$
D_{\tau}^{-1-\beta}\left\|\mathfrak{\Im}_{x} f\right\|_{L(\Omega)}^{2} \leq \frac{T^{\beta}}{\Gamma(1+\beta)} \int_{0}^{\tau}\left\|\mathfrak{\Im}_{x} f\right\|_{L(\Omega)}^{2} d t,
$$

we reduce inequality (34) as follows

$$
\begin{aligned}
& D_{\tau}^{\beta-1}\left\|\Im_{x} \frac{\partial v}{\partial t}\right\|_{L^{2}(\Omega)}^{2}+\int_{0}^{\tau}\left\|\frac{\partial v}{\partial t}(., t)\right\|_{L^{2}(\Omega)}^{2} d t+\|v(., \tau)\|_{L^{2}(\Omega)}^{2} \\
\leq & \delta_{7}\left\{\int_{0}^{T}\left\|\Im_{x} f(., t)\right\|_{L(\Omega)}^{2} d t+\|\varphi\|_{L^{2}(\Omega)}^{2}+\left\|\Im_{x} \psi\right\|_{L^{2}(\Omega)}^{2}\right\} .
\end{aligned}
$$




$$
\delta_{7}=\delta_{6}\left(1+\frac{T^{\beta}}{\Gamma(1+\beta)}\right)
$$

Since the RHS of estimate (36) is independent of $\tau$, we can take the supremum on the LHS with respect to $\tau$ over $[0, T]$. Thus, we get the desired inequality (19). Theorem (4) proof is complete.

\section{Existence of the linear problem solution}

The current section's aim is to prove the existence of the strong solution of problems (6)-(8). It remains to demonstrate the density of the range $R(L)$.

Proposition 5. [32] The operator L engendered by problems (1)-(3) has a closure.

Defining the operator equation solution

$$
\bar{L} v=\mathcal{F}=(f, \varphi, \psi),
$$

as a strong solution of problems (6)-(8). The inequality (19) can be extended into

$$
\|v\|_{E} \leq\|\bar{L} v\|_{F}, \quad \forall v \in D(\bar{L}) .
$$

the inequality demonstrated above assures the strong solution uniqueness.

Corollary 6. The range of the operator $\bar{L}$ is closed in $F$ and $R(\bar{L})=\overline{R(L)}$ and $\bar{L}^{-1}=\overline{L^{-1}}$.

Theorem 7. Let Theorem (4) conditions be verified. Then, for any $\mathcal{F}=(f, g, h) \in F$, the problems (6)-(8) have a unique solution $v$ such that $v=\bar{L}^{-1} \mathcal{F}=\overline{L^{-1}} \mathcal{F}$.

Proposition 8. Let Assumption (1) be fulfilled. If for a certain function $g \in L^{2}(Q)$, and every $v \in D(L)$ verifying homogenous initial conditions, we have

$$
(\mathcal{L} v, g)_{L^{2}(D)}=0
$$

then $g$ vanishes almost everywhere in D are as follows:

Proof. Introducing a new function $\sigma(x, t)$ verifies conditions (2) and (3), and $\sigma, \sigma_{x}, \mathfrak{s}_{t} \sigma_{x}, \mathfrak{s}_{t} \sigma$ and ${ }^{C} \partial_{t}^{\beta+1} \sigma \in L^{2}(D)$, then we pose

$$
v(x, t)=\Im_{t}^{2} \sigma,
$$

where

$$
\Im_{t} \sigma=\int_{0}^{t} \sigma(x, s) d s, \quad \Im_{t}^{2} \sigma=\int_{0}^{t} \int_{0}^{s} \sigma(x, z) d z d s
$$

Equation (38) then becomes

$$
\begin{aligned}
& \left({ }^{c} \partial_{t}^{\beta+1} \mathfrak{\Im}_{t}^{2} \sigma-\frac{\partial}{\partial x}\left(\gamma(x, t) \mathfrak{\Im}_{t}^{2}\left(\frac{\partial \sigma}{\partial x}\right)\right)-\frac{\partial^{2}}{\partial x \partial t}\left(\eta(x, t) \mathfrak{\Im}_{t}^{2}\left(\frac{\partial \sigma}{\partial x}\right)\right)-\int_{0}^{t} \xi(t-z) \mathfrak{\Im}_{t}^{2} \sigma(x, z) d z, g\right)_{L^{2}(D)} \\
& =0 .
\end{aligned}
$$


Now, we consider the function

$$
g(x, t)=-\mathfrak{\Im}_{t} \mathfrak{\Im}_{x}^{2} \sigma .
$$

Obviously, the function $g$ included in $L^{2}(D)$. Equations (39)-(40) lead to

$$
\begin{aligned}
& -\left({ }^{c} \partial_{t}^{\beta+1} \mathfrak{\Im}_{t}^{2} \sigma, \mathfrak{\Im}_{t} \mathfrak{\Im}_{x}^{2} \sigma\right)_{L^{2}(D)}+\left(\frac{\partial}{\partial x}\left(\gamma(x, t) \mathfrak{\Im}_{t}^{2}\left(\frac{\partial \sigma}{\partial x}\right)\right), \mathfrak{\Im}_{t} \mathfrak{\Im}_{x}^{2} \sigma\right)_{L^{2}(D)} \\
& +\left(\frac{\partial^{2}}{\partial x \partial t}\left(\eta(x, t) \mathfrak{\Im}_{t}^{2}\left(\frac{\partial \sigma}{\partial x}\right)\right), \mathfrak{\Im}_{t} \mathfrak{\Im}_{x}^{2} \sigma\right)_{L^{2}(D)}+\left(\int_{0}^{t} \xi(t-z) \mathfrak{\Im}_{t}^{2} \sigma(x, z) d z, \mathfrak{\Im}_{t} \mathfrak{\Im}_{x}^{2} \sigma\right)_{L^{2}(D)} \\
= & 0
\end{aligned}
$$

Note that the function $\sigma$ verifies conditions (2)-(3), then we have

$$
\begin{aligned}
-\left({ }^{C} \partial_{t}^{\beta+1} \mathfrak{\Im}_{t}^{2} \sigma, \mathfrak{\Im}_{t} \mathfrak{\Im}_{x}^{2} \sigma\right)_{L^{2}(D)} & =\left({ }^{C} \partial_{t}^{\beta} \mathfrak{\Im}_{x} \mathfrak{\Im}_{t} \sigma, \mathfrak{\Im}_{x} \mathfrak{\Im}_{t} \sigma\right)_{L^{2}(D)} \\
\left(\frac{\partial}{\partial x}\left(\gamma(x, t) \mathfrak{\Im}_{t}^{2}\left(\frac{\partial \sigma}{\partial x}\right)\right), \mathfrak{\Im}_{t} \mathfrak{\Im}_{x}^{2} \sigma\right)_{L^{2}(D)}= & \frac{1}{2} \int_{0}^{1} \gamma\left(\mathfrak{\Im}_{t}^{2} \sigma\right)^{2} d x-\frac{1}{2} \int_{D} \frac{\partial \gamma}{\partial t}\left(\mathfrak{\Im}_{t}^{2} \sigma\right)^{2} d x d t \\
& +\left(\frac{\partial \gamma(x, t)}{\partial x}\left(\mathfrak{\Im}_{t}^{2} \sigma\right), \mathfrak{\Im}_{t} \mathfrak{\Im}_{x} \sigma\right)_{L^{2}(D)} \\
\left(\frac{\partial^{2}}{\partial x \partial t}\left(\eta(x, t) \mathfrak{\Im}_{t}^{2}\left(\frac{\partial \sigma}{\partial x}\right)\right), \mathfrak{\Im}_{t} \mathfrak{\Im}_{x}^{2} \sigma\right)_{L^{2}(D)}= & \left(\frac{\partial^{2} \eta}{\partial x \partial t} \mathfrak{\Im}_{t}^{2} \sigma, \mathfrak{\Im}_{t} \mathfrak{\Im}_{x} \sigma\right)_{L^{2}(D)}+\left(\frac{\partial \eta}{\partial t} \mathfrak{\Im}_{t}^{2} \sigma, \mathfrak{\Im}_{t} \sigma\right)_{L^{2}(D)} \\
& +\int_{D} \frac{\partial^{2} \eta}{\partial x^{2}}\left(\mathfrak{\Im}_{t} \mathfrak{\Im}_{x} \sigma\right)^{2} d x d t+\int_{D} \eta\left(\mathfrak{\Im}_{t} \sigma\right)^{2} d x d t
\end{aligned}
$$

Insertion of Equations (42)-(44) into (41), yields

$$
\begin{aligned}
& -2\left({ }^{C} \partial_{t}^{\beta} \mathfrak{\Im}_{x} \mathfrak{\Im}_{t} \sigma, \mathfrak{\Im}_{x} \mathfrak{\Im}_{t} \sigma\right)_{L^{2}(D)}+\int_{0}^{1} \gamma\left(\mathfrak{\Im}_{t}^{2} \sigma\right)^{2} d x=\int_{D} \frac{\partial \gamma}{\partial t}\left(\mathfrak{\Im}_{t}^{2} \sigma\right)^{2} d x d t \\
& -2 \int_{D} \frac{\partial^{2} \eta}{\partial x^{2}}\left(\mathfrak{\Im}_{t} \mathfrak{\Im}_{x} \sigma\right)^{2} d x d t-2 \int_{D} \eta\left(\mathfrak{\Im}_{t} \sigma\right)^{2} d x d t-2\left(\left(\frac{\partial^{2} \eta}{\partial x \partial t}+\frac{\partial \gamma(x, t)}{\partial x}\right) \mathfrak{\Im}_{t}^{2} \sigma, \mathfrak{\Im}_{t} \mathfrak{\Im}_{x} \sigma\right)_{L^{2}(D)} \\
& -2\left(\frac{\partial \eta}{\partial t} \Im_{t}^{2} \sigma, \mathfrak{\Im}_{t} \sigma\right)_{L^{2}(D)}-2\left(\int_{0}^{t} \xi(t-z) \mathfrak{\Im}_{s}^{2} \sigma(x, z) d z, \mathfrak{\Im}_{t} \mathfrak{\Im}_{x}^{2} \sigma\right)_{L^{2}(D)}
\end{aligned}
$$

According to Lemma 1, we bound the first term on the LHS of (45); we have

$$
2\left({ }^{C} \partial_{t}^{\beta} \mathfrak{\Im}_{x} \mathfrak{\Im}_{t} \sigma, \mathfrak{\Im}_{x} \mathfrak{\Im}_{t} \sigma\right)_{L^{2}(D)} \geq{ }^{C} \partial_{t}^{\beta}\left\|\mathfrak{\Im}_{x} \mathfrak{\Im}_{t} \sigma\right\|_{L^{2}(D)}^{2},
$$

Also, we bound the last three terms on the RHS of (45) utilizing inequality 17, and we then get 


$$
\begin{gathered}
2\left(\left(\frac{\partial \gamma(x, t)}{\partial x}+\frac{\partial^{2} \eta}{\partial x \partial t}\right) \mathfrak{\Im}_{t}^{2} \sigma, \Im_{t} \Im_{x} \sigma\right)_{L^{2}(D)} \leq 2\left(c_{3}^{2}+c_{9}^{2}\right)\left\|\Im_{\Im_{t}}^{2} \sigma\right\|_{L^{2}(D)}^{2}+\left\|\Im_{x} \Im_{t} \sigma\right\|_{L^{2}(D)}^{2} \\
2\left(\frac{\partial \eta}{\partial t}\left(\Im_{t}^{2} \sigma\right), \Im_{t} \sigma\right)_{L^{2}(D)} \leq 2 c_{4}\left\|\Im_{t}^{2} \sigma\right\|_{L^{2}(D)}^{2}+\frac{c_{6}^{2}}{2 c_{4}^{2}}\left\|\Im_{t} \sigma\right\|_{L^{2}(D)}^{2} \\
\left(\int_{0}^{t} \xi(t-z) \mathfrak{\Im}_{z}^{2} \sigma(x, z) d z, \Im_{x^{2}}^{2} \Im_{t} \sigma\right)_{L^{2}(D)} \leq c_{10} T^{2}\left\|\Im_{t}^{2} \sigma\right\|_{L(D)}^{2}+\frac{1}{2}\left\|\Im_{x} \Im_{t} \sigma\right\|_{L(D)}^{2}
\end{gathered}
$$

The insertion of estimates (46)-(48) in Equation (45) gives

$$
D_{\tau}^{\beta-1}\left\|\mathfrak{\Im}_{x} \Im_{t} \sigma\right\|_{L^{2}(D)}^{2}+\int_{0}^{1}\left(\mathfrak{\Im}_{t}^{2} \sigma\right)^{2} d x \leq \delta_{8}\left(\int_{D}\left(\mathfrak{\Im}_{t}^{2} \sigma\right)^{2} d x d t+\left\|\Im_{x} \Im_{\Im_{t}} \sigma\right\|_{L^{2}(D)}^{2}\right)
$$

with

$$
\delta_{8}=\frac{\max \left(c_{2}+2\left(c_{3}^{2}+c_{9}^{2}\right)+c_{10} T^{2}+\frac{c_{6}^{2}}{2 c_{4}}, 2 c_{10}+\frac{3}{2}\right)}{\min \left(1, c_{0}\right)}
$$

Eliminating the first term on the LHS of (50), using Lemma 2, with

$$
S(\tau)=\int_{0}^{\tau} \int_{0}^{1}\left(\Im_{t}^{2} \sigma\right)^{2} d x d t
$$

observe that $S(0)=0$, then we get

$$
S(\tau) \leq T \exp \left(T \delta_{8}\right)\left\|\mathfrak{I}_{x} \mathfrak{\Im}_{t} \sigma\right\|_{L^{2}\left(D^{\tau}\right)}^{2},
$$

Similarly, by discarding the second integral on the LHS of (50) and applying (53), we obtain

$$
D_{\tau}^{\beta-1}\left\|\mathfrak{\Im}_{x} \mathfrak{\Im}_{t} \sigma\right\|_{L^{2}(D)}^{2} \leq \delta_{8}\left(T \exp \left(T \delta_{8}\right)+1\right)\left\|\mathfrak{\Im}_{x} \mathfrak{\Im}_{t} \sigma\right\|_{L^{2}(D)}^{2}
$$

by Lemma 2 , with

$$
S(\tau)=\int_{0}^{\tau} \int_{0}^{1}\left(\Im_{x} \Im_{\Im_{t}} \sigma\right)^{2} d x d t
$$

and

$$
{ }^{c} \partial_{\tau}^{\beta} S(\tau)=D_{\tau}^{\beta-1}\left\|\mathfrak{I}_{x} \widetilde{\Im}_{t} \sigma\right\|_{L^{2}(D)}^{2}, S(0)=0
$$

it follows that

$$
\begin{aligned}
& \left\|\Im_{x} \Im_{\Im_{t}} \sigma\right\|_{L^{(2}\left(D^{\tau}\right)}^{2} \leq S(0) E_{\beta}\left(\delta_{8}\left(T \exp \left(T \delta_{8}\right)+1\right) \tau^{\beta}\right)+\Gamma(\beta) E_{\beta, \beta}\left(\delta_{8}\left(T \exp \left(T \delta_{8}\right)+1\right) \tau^{\beta}\right) D_{\tau}^{-\beta}(0) \\
= & 0
\end{aligned}
$$

for any $\tau \in[0, T]$. Hence inequality (55) shows that $g=0$ ae in $D$. Continuing Theorem 7 proof, we assume that for a certain function $G=\left(g, g_{0}, g_{1}\right) \in R(L)^{\perp}$, we have 


$$
(\mathcal{L} v, g)_{L^{2}(D)}+\left(l_{1} v, g_{0}\right)_{L^{2}(\Omega)}+\left(l_{2} v, g_{1}\right)_{L^{2}(\Omega)}=0
$$

then we should show that $g_{0}=0, g_{1}=0$. Putting $v \in D(L)$, verifying homogenous initial conditions into (56), yields

$$
(\mathcal{L} v, g)_{L^{2}(D)}=0, \forall v \in D(L)
$$

By applying Proposition (8) to (57), we see that $g=0$. Consequently, (56) becomes

$$
\left(l_{1} v, g_{0}\right)_{L^{2}(\Omega)}+\left(l_{2} v, g_{1}\right)_{L^{2}(\Omega)}=0 \forall v \in D(L),
$$

Since $l_{1} v$ and $l_{2} v$ are independent and their ranges $l_{1}$ and $l_{2}$ are everywhere dense in $L^{2}(\Omega)$, we conclude that $g_{0}=g_{1}=0$, this complete the proof of Theorem 7 .

\section{The study of the nonlinear problem}

This section is devoted to solving the main problems (1)-(3). Consider now the auxiliary problem with the homogenous equation:

$$
\begin{gathered}
\mathcal{L} V={ }^{C} \partial_{t}^{\beta+1} V-\frac{\partial}{\partial x}\left(\gamma(x, t) \frac{\partial V}{\partial x}\right)-\frac{\partial^{2}}{\partial x \partial t}\left(\eta(x, t) \frac{\partial V}{\partial x}\right)-\int_{0}^{t} \xi(t-z) V(x, z) d z=0 \\
\ell_{1} V=V(x, 0)=0, \quad \ell_{2} V=\frac{\partial V(x, 0)}{\partial t}=0, \quad x \in \Omega, \\
\int_{0}^{1} V(x, t) d x=0, \quad V_{x}(1, t)=0, \quad t \in(0, T),
\end{gathered}
$$

If $V$ and $v$ are solutions of problems (8)-(6),(1)-(3), respectively, then $h=v-V$ satisfies

$$
\begin{gathered}
\mathcal{L} h={ }^{C} \partial_{t}^{\beta+1} h-\frac{\partial}{\partial x}\left(\gamma(x, t) \frac{\partial h}{\partial x}\right)-\frac{\partial^{2}}{\partial x \partial t}\left(\eta(x, t) \frac{\partial h}{\partial x}\right)-\int_{0}^{t} \xi(t-z) h(x, z) d z \\
=G\left(x, t, h, \frac{\partial h}{\partial x}\right) \\
\ell_{1} h=h(x, 0)=0, \quad \ell_{2} h=\frac{\partial h}{\partial t}(x, 0)=0, \quad x \in \Omega, \\
\int_{0}^{1} h(x, t) d x=0, \quad h_{x}(1, t)=0, \quad t \in(0, T),
\end{gathered}
$$

such that the function $G\left(x, t, h, \frac{\partial h}{\partial x}\right)=f\left(x, t, h+V, \frac{\partial h}{\partial x}+\frac{\partial V}{\partial x}\right)$, verifies the following
condition

$$
\left|G\left(x, t, w_{1}, y_{1}\right)-G\left(x, t, w_{2}, y_{2}\right)\right| \leq L\left(\left|w_{1}-w_{2}\right|+\left|y_{1}-y_{2}\right|\right) \quad \text { forall }(x, t) \in D .
$$

Theorem 7 shows that the solution of problems (6)-(8) is unique and depends continuously on the initial data. It remains to establish a similar proof for problems (62)-(64). We introduce the space 


$$
\tilde{C}^{1}(D)=\left\{w \in C^{1}(D) \quad \text { such that, } \quad \frac{\partial w^{2}}{\partial t \partial x} \in C(D)\right\}
$$

Suppose that $h$ and $u \in \tilde{C}^{1}(D)$ verify homogenous initial and boundary conditions $h(x, T)=0, h(x, 0)=0, \int_{0}^{1} w(x, t) d x=0$. For $u \in \tilde{C}^{1}(D)$, we have

$$
\begin{aligned}
& \left(\mathcal{L} h, \mathfrak{\Im}_{x} u\right)_{L^{2}(D)}=\left({ }^{C} \partial_{t}^{\beta+1} h, \mathfrak{\Im}_{x} u\right)_{L^{2}(D)}-\left(\frac{\partial}{\partial x}\left(\gamma(x, t) \frac{\partial h}{\partial x}\right), \mathfrak{\Im}_{x} u\right)_{L^{2}(D)} \\
& -\left(\frac{\partial^{2}}{\partial x \partial t}\left(\eta(x, t) \frac{\partial h}{\partial x}\right), \mathfrak{\Im}_{x} u\right)_{L^{2}(D)}-\left(\int_{0}^{t} \xi(t-z) h(x, z) d z, \Im_{x} u\right)_{L^{2}(D)}
\end{aligned}
$$

Computation of all terms of Equation (67), using conditions on $h$ and $u$, gives

$$
\begin{gathered}
\left({ }^{C} \partial_{t}^{\beta+1} h, \Im_{x} u\right)_{L^{2}(D)}=-\left({ }^{C} \partial_{t}^{\beta+1} \Im_{x} h, u\right)_{L^{2}(D)} \\
-\left(\frac{\partial}{\partial x}\left(\gamma \frac{\partial h}{\partial x}\right), \Im_{x} u\right)_{L^{2}(D)}=\left(\gamma \frac{\partial h}{\partial x}, u\right)_{L^{2}(D)} \\
-\left(\frac{\partial^{2}}{\partial x \partial t}\left(\eta \frac{\partial h}{\partial x}\right), \Im_{x} u\right)_{L^{2}(D)}=\left(\frac{\partial}{\partial t}\left(\eta \frac{\partial h}{\partial x}\right), u\right)_{L^{2}(D)} \\
-\left(\int_{0}^{t} \xi(t-z) h(x, z) d z, \mathfrak{\Im}_{x} u\right)_{L^{2}(D)}=\left(\int_{0}^{t} \xi(t-z) \mathfrak{\Im}_{x} h(x, z) d z, u\right)_{L^{2}(D)}
\end{gathered}
$$

Insertion of (68)-(71) into (67) yields

$$
R(h, u)=\left(u, \Im_{x} G\right)_{L^{2}(D)}
$$

such that

$$
\begin{aligned}
R(h, u)= & -\left({ }^{C} \partial_{t}^{\beta+1} \widetilde{\Im}_{x} h, u\right)_{L^{2}(D)}+\left(\gamma \frac{\partial h}{\partial x}, u\right)_{L^{2}(D)}+\left(\frac{\partial}{\partial t}\left(\eta \frac{\partial h}{\partial x}\right), u\right)_{L^{2}(D)} \\
& +\left(\int_{0}^{t} \xi(t-z) \mathfrak{\Im}_{x} h(x, z) d z, u\right)_{L^{2}(D)}
\end{aligned}
$$

Definition 9. A function $h \in L^{2}\left(0, T, H^{1}(\Omega)\right)$ is considered as the problems (62)-(64) weak solution if it satisfies (64) and (72) holds.

Constructing an iteration sequence as follows: let $h(0)=0$, and let defining the sequence $\left(h^{(n)}\right)_{n} \in \mathbb{N}$ as follows: if $h^{(n-1)}$ is given, then for $n \in \mathbb{N}$ solve the following problem:

$$
\begin{aligned}
\mathcal{L} h & ={ }^{C} \partial_{t}^{\beta+1} h^{(n)}-\frac{\partial}{\partial x}\left(\gamma \frac{\partial h^{(n)}}{\partial x}\right)-\frac{\partial^{2}}{\partial x \partial t}\left(\eta \frac{\partial h^{(n)}}{\partial x}\right)-\int_{0}^{t} \xi(t-z) h^{(n)}(x, z) d z \\
& =G\left(x, t, h^{(n-1)}, \frac{\partial h^{(n-1)}}{\partial x}\right)
\end{aligned}
$$




$$
\begin{gathered}
\ell_{1} h^{(n)}=h^{(n)}(x, 0)=0, \quad \ell_{2} h^{(n)}=\frac{\partial h^{(n)}}{\partial t}(x, 0)=0, \quad x \in \Omega, \\
\int_{0}^{1} h^{(n)}(x, t) d x=0, \quad h_{x}^{(n)}(1, t)=0, \quad t \in(0, T),
\end{gathered}
$$

Solvability of nonlinear fractional equation

Theorem 10. For each fixed n, assume that the solution of problems (74)-(76) $h^{(n)}(x, t)$ is unique. If we put $H^{(n)}(x, t)=h^{(n+1)}(x, t)-h^{(n)}(x, t)$, then we obtain

$$
\begin{gathered}
\mathcal{L} H^{(n)}={ }^{C} \partial_{t}^{\beta+1} H^{(n)}-\frac{\partial}{\partial x}\left(\gamma \frac{\partial H^{(n)}}{\partial x}\right)-\frac{\partial^{2}}{\partial x \partial t}\left(\eta \frac{\partial H^{(n)}}{\partial x}\right)-\int_{0}^{t} \xi(t-z) H^{(n)}(x, z) d z \\
=\Psi^{(n-1)}(x, t) \\
\ell_{1} H^{(n)}=H^{(n)}(x, 0)=0, \quad \ell_{2} H^{(n)}=\frac{\partial H^{(n)}(x, 0)}{\partial t}=0, \quad x \in \Omega, \\
\int_{0}^{1} H^{(n)}(x, t) d x=0, \quad H_{x}^{(n)}(1, t)=0, \quad t \in(0, T),
\end{gathered}
$$

with

$$
\Psi^{(n-1)}(x, t)=G\left(x, t, h^{(n)}, \frac{\partial h^{(n)}}{\partial x}\right)-G\left(x, t, h^{(n-1)}, \frac{\partial h^{(n-1)}}{\partial x}\right)
$$

Lemma 11. Under Assumptions (1), and supposing that the condition (65) holds, then for the linearized problems (77)-(79), the following estimate holds

$$
\left\|H^{(n)}\right\|_{L^{2}\left(0, T, H^{1}(\Omega)\right)} \leq K\left\|H^{(n-1)}\right\|_{L^{2}\left(0, T, H^{1}(\Omega)\right)}
$$

where $K>0$ is constant given by

$$
K=\exp \left(\delta_{10} T\right)\left(1+\Gamma(\beta) E_{\beta, \beta}\left(\delta_{9} \exp \left(\delta_{10} T\right) t^{\beta}\right) \frac{T^{\beta}}{\Gamma(1+\beta)}\right)
$$

Proof. We take the scalar product in $L^{2}\left(D^{\tau}\right), \tau \in[0, T]$ of (77) and the integro-differential operator $M H^{(n)}=-\Im_{x}^{2} H^{(n)}$, we get

$$
\begin{aligned}
& 2\left({ }^{C} \partial_{t}^{\beta+1} H^{(n)},-\Im_{x}^{2} \frac{\partial H^{(n)}}{\partial t}\right)_{L^{2}\left(D^{\tau}\right)}-2\left(\frac{\partial}{\partial x}\left(\gamma(x, t) \frac{\partial H^{(n)}}{\partial x}\right),-\Im_{x}^{2} \frac{\partial H^{(n)}}{\partial t}\right)_{L^{2}\left(D^{\tau}\right)} \\
& -2\left(\frac{\partial^{2}}{\partial x \partial t}\left(\eta(x, t) \frac{\partial H^{(n)}}{\partial x}\right),-\Im_{x}^{2} \frac{\partial H^{(n)}}{\partial t}\right)_{L^{2}\left(D^{\tau}\right)} \\
& -2\left(\int_{0}^{t} \xi(t-z) H^{(n)}(x, z) d z,-\Im_{x}^{2} \frac{\partial H^{(n)}}{\partial t}\right)_{L^{2}\left(D^{\tau}\right)} \\
& =2\left(\Psi^{(n-1)}(x, t),-\Im_{x}^{2} \frac{\partial H^{(n)}}{\partial t}\right)_{L^{2}\left(D^{\tau}\right)} .
\end{aligned}
$$


Integrations by parts all terms of (81), by using conditions (78)-(79), proceeding as in the establishment of Theorem 4, yields

$$
\begin{aligned}
& D^{\beta-1}\left\|\Im_{x} \frac{\partial H^{(n)}}{\partial t}\right\|_{L^{2}(\Omega)}^{2}+\left(c_{0}+c_{5}\right)\left\|H^{(n)}(., \tau)\right\|_{L^{2}(\Omega)}^{2} \leq \int_{0}^{\tau}\left\|\Im_{x} \Psi^{(n-1)}(x, t)\right\|_{L(\Omega)}^{2} d t \\
& +\left(\frac{5}{2}+\frac{c_{7}^{2}}{2 c_{4}}\right) \int_{0}^{\tau}\left\|\Im_{x} \frac{\partial H^{(n)}}{\partial t}(., t)\right\|_{L(\Omega)}^{2} d t \\
& +\left(2\left(c_{3}^{2}+c_{9}^{2}\right)+c_{2}+c_{8}+c_{10} T^{2}\right) \int_{0}^{\tau}\left\|H^{(n)}(., t)\right\|_{L^{2}(\Omega)}^{2} d t
\end{aligned}
$$

On the other hand, applying to Equation (77) the operator $\mathfrak{\Im}_{x}$, and taking into consideration condition (79), multiplying the resulting equation with $\frac{\partial T^{(n)}}{\partial x}$ and integrating over $D^{\tau}$, we get

$$
\begin{aligned}
& \int_{D^{\tau}}{ }^{c} \partial_{t}^{\beta+1} \Im_{x} H^{(n)} \frac{\partial H^{(n)}}{\partial x} d x d t-\int_{D^{\tau}} \gamma(x, t)\left(\frac{\partial H^{(n)}}{\partial x}\right)^{2} d x d t-\int_{D^{\tau}} \frac{\partial}{\partial t}\left(\eta(x, t) \frac{\partial H^{(n)}}{\partial x}\right) \frac{\partial H^{(n)}}{\partial x} d x d t \\
& -\int_{D^{\tau}} \int_{0}^{t} \xi(t-z) \mathfrak{\Im}_{x} H^{(n)}(x, z) \frac{\partial H^{(n)}}{\partial x} d z d x d t=\int_{D^{\tau}} \mathfrak{\Im}_{x} \Psi^{(n-1)}(x, t) \frac{\partial H^{(n)}}{\partial x} d x d t
\end{aligned}
$$

After integration by parts of all the terms of (83) and taking into consideration conditions (78), (79) and using inequality (17), we have

$$
\begin{aligned}
& \int_{D^{\tau}}{ }^{c} \partial_{t}^{\beta+1} H^{(n)} H^{(n)} d x d t+c_{0} \int_{0}^{\tau}\left\|\frac{\partial H^{(n)}}{\partial x}(., t)\right\|_{L^{2}(\Omega)} d t+\frac{1}{2} c_{4}\left\|\frac{\partial H^{(n)}}{\partial x}(., \tau)\right\|_{L^{2}(\Omega)}^{2} \\
\leq & \frac{1}{2} \int_{0}^{\tau}\left\|\Psi^{(n-1)}\right\|_{L^{2}(\Omega)} d t+\left(c_{10} T^{2}+1\right) \int_{0}^{\tau}\left\|H^{(n)}(., t)\right\|_{L^{2}(\Omega)} d t
\end{aligned}
$$

Combination of inequalities (83)-(84) gives

$$
\begin{aligned}
& D_{\tau}^{\beta-1}\left\|\Im_{x} \frac{\partial H^{(n)}}{\partial t}\right\|_{L^{2}(\Omega)}+\int_{0}^{\tau} \partial_{t}^{\beta+1} H^{(n)} H^{(n)} d x d t+c_{0} \int_{0}^{\tau}\left\|\frac{\partial H^{(n)}}{\partial x}(., t)\right\|_{L^{2}(\Omega)} d t+\frac{1}{2} c_{4}\left\|\frac{\partial H^{(n)}}{\partial x}(., \tau)\right\|_{L^{2}(\Omega)}^{2} \\
& +\left(c_{0}+c_{5}\right)\left\|H^{(n)}(., \tau)\right\|_{L^{2}(\Omega)} \leq \int_{0}^{\tau}\left\|\Psi^{(n-1)}\right\|_{L^{2}(\Omega)} d t+\left(\frac{5}{2}+\frac{C_{7}^{2}}{2 C_{4}}\right) \int_{0}^{\tau}\left\|\Im_{x} \frac{\partial H^{(n)}}{\partial t}(., t)\right\|_{L^{2}(\Omega)} d t \\
& +\left(\left(2\left(c_{3}^{2}+c_{9}^{2}\right)+c_{2}+c_{8}+c_{10} T^{2}+1\right) \int_{0}^{\tau}\left\|H^{(n)}(., t)\right\|_{L^{2}(\Omega)}^{2} d t\right.
\end{aligned}
$$

Eliminating the last term on the RHS of (85), by using Gronwall's lemma, it comes 


$$
\begin{aligned}
& D_{\tau}^{\beta-1}\left\|\Im_{x} \frac{\partial H^{(n)}}{\partial t}\right\|_{L^{2}(\Omega)}+\int_{0}^{\tau} \partial_{t}^{\beta+1} H^{(n)} H^{(n)} d x d t+\left(c_{0}+c_{5}\right)\left\|H^{(n)}(., \tau)\right\|_{L^{2}(\Omega)} \\
& +\frac{1}{2} c_{4}\left\|\frac{\partial H^{(n)}}{\partial x}(., \tau)\right\|_{L^{2}(\Omega)}^{2}+c_{0} \int_{0}^{\tau}\left\|\frac{\partial H^{(n)}}{\partial x}(., t)\right\|_{L^{2}(\Omega)} d t \\
\leq & \exp \left(\delta_{10}\right)\left\{\int_{0}^{\tau}\left\|\Psi^{(n-1)}\right\|_{L(\Omega)}^{2} d t+\delta_{9} \int_{0}^{\tau}\left\|\Im_{x} \frac{\partial H^{(n)}}{\partial t}(., t)\right\|_{L(\Omega)}^{2} d t\right\}
\end{aligned}
$$

Solvability of nonlinear fractional

where

$$
\begin{aligned}
& \delta_{9}=\frac{5}{2}+\frac{C_{7}^{2}}{2 C_{4}} \\
& \delta_{10}=2\left(c_{3}^{2}+c_{9}^{2}\right)+c_{2}+c_{8}+c_{10} T^{2}+1
\end{aligned}
$$

To discard the last integral on the RHS of inequality (86), we drop the three first elements then use the Gronwall's lemma, it follows

$$
\int_{0}^{\tau}\left\|\mathfrak{I}_{x} \frac{\partial H^{(n)}}{\partial t}\right\|_{L^{2}(\Omega)}^{2} d t \leq \Gamma(\beta) E_{\beta, \beta}\left(\delta_{9} \exp \left(\delta_{10} T\right) t^{\beta}\right) \exp \left(\delta_{9} t\right) D_{t}^{-\beta}\left\|\Psi^{(n-1)}\right\|_{L(\Omega)}^{2}
$$

On the other hand, via the condition (65), we get

$$
\int_{0}^{\tau}\left\|\Psi^{(n-1)}\right\|_{L(\Omega)}^{2} d t \leq 2 L^{2} \int_{0}^{\tau}\left(\left\|H^{(n-1)}(., t)\right\|_{L^{2}(\Omega)}^{2}+\left\|\frac{\partial H^{(n-1)}(., t)}{\partial x}\right\|_{L^{2}(\Omega)}^{2}\right) d t
$$

Combining (86)-(88) and by using (35), we get

$$
\begin{aligned}
& D^{\beta-1}\left\|\Im_{x} \frac{\partial H^{(n)}}{\partial t}\right\|_{L^{2}(\Omega)}^{2}+\int_{0}^{\tau} \partial_{t}^{\beta+1} H^{(n)} H^{(n)} d x d t+\int_{0}^{\tau}\left\|\frac{\partial H^{(n)}}{\partial x}(., t)\right\|_{L^{2}(\Omega)}^{2} d t+\left\|\frac{\partial H^{(n)}}{\partial x}(., \tau)\right\|_{L^{2}(\Omega)}^{2} \\
& +\left\|H^{(n)}(., \tau)\right\|_{L^{2}(\Omega)}^{2} \leq \delta_{11} L^{2} \int_{0}^{T}\left(\left\|H^{(n-1)}(., t)\right\|_{L^{2}(\Omega)}^{2}+\left\|\frac{\partial H^{(n-1)}}{\partial x}(., t)\right\|_{L^{2}(\Omega)}^{2}\right) d t
\end{aligned}
$$

where

$$
\delta_{11}=\exp \left(\delta_{10} T\right)\left(1+\Gamma(\beta) E_{\beta, \beta}\left(\delta_{9} \exp \left(\delta_{10} T\right) t^{\beta}\right) \frac{T^{\beta}}{\Gamma(1+\beta)}\right)
$$

After discarding the first two terms on the LHS of inequality (89), we get

$$
\begin{aligned}
& \int_{0}^{\tau}\left\|\frac{\partial H^{(n)}}{\partial x}(., t)\right\|_{L^{2}(\Omega)}^{2} d t+\left\|H^{(n)}(., \tau)\right\|_{L^{2}(\Omega)}^{2}+\left\|\frac{\partial H^{(n)}}{\partial x}(., \tau)\right\|_{L^{2}(\Omega)}^{2} \\
\leq & \delta_{11} L^{2} \int_{0}^{T}\left(\left\|H^{(n-1)}(., t)\right\|_{L^{2}(\Omega)}^{2}+\left\|\frac{\partial H^{(n-1)}}{\partial x}(., t)\right\|_{L^{2}(\Omega)}^{2}\right) d t
\end{aligned}
$$

Here, the RHS doesn't depend on $\tau$ so, we can replace the LHS by upper bounds with respect to $\tau$, we obtain 
AJMS

$$
\begin{aligned}
& \int_{0}^{T}\left\|\frac{\partial H^{(n)}}{\partial x}(., t)\right\|_{L^{2}(\Omega)}^{2} d t+\left\|H^{(n)}(., \tau)\right\|_{L^{2}(\Omega)}^{2}+\left\|\frac{\partial H^{(n)}}{\partial x}(., \tau)\right\|_{L^{2}(\Omega)}^{2} \\
\leq & \delta_{11} L^{2} \int_{0}^{T}\left(\left\|H^{(n-1)}(., t)\right\|_{L^{2}(\Omega)}^{2}+\left\|\frac{\partial H^{(n-1)}}{\partial x}(., t)\right\|_{L^{2}(\Omega)}^{2}\right) d t
\end{aligned}
$$

Now, we integrate over $(0, T)$, we get

$$
\begin{gathered}
\int_{0}^{T}\left\|H^{(n)}(., \tau)\right\|_{L^{2}(\Omega)}^{2} d t+\int_{0}^{T}\left\|\frac{\partial H^{(n)}}{\partial x}(., t)\right\|_{L^{2}(\Omega)}^{2} d t \\
\leq \delta_{12} L^{2} \int_{0}^{T}\left(\left\|H^{(n-1)}(., t)\right\|_{L^{2}(\Omega)}^{2}+\left\|\frac{\partial H^{(n-1)}}{\partial x}(., t)\right\|_{L^{2}(\Omega)}^{2}\right) d t \\
\delta_{12}=\frac{\delta_{11} L^{2} T}{\min (1, T)}
\end{gathered}
$$

We get then the desired inequality (80).

$$
\left\|H^{(n)}\right\|_{L^{2}\left(0, T, H^{1}(\Omega)\right)} \leq \delta_{12}\left\|H^{(n-1)}\right\|_{L\left(0, T, H^{1}(\Omega)\right)}
$$

Using the convergence of series criteria we conclude that $\sum_{n=1}^{\infty} H^{(n)}$ converges if $\frac{\delta_{11} L^{2} T}{\min (1, T)}<1$, namely if $L<\sqrt{\frac{\min (1, T)}{\delta_{11} T}}$. Since $H^{(n)}(x, t)=h^{(n+1)}(x, t)-h^{(n)}(x, t)$, then the sequence $\left(h^{(n)}\right)_{n \in \mathbb{N}}$ given by $h^{(n)}(x, t)=\sum_{i=0}^{n-1} H^{(i)}+h^{(0)}(x, t), i \in \mathbb{N}$ converges to a function $h \in L^{2}\left((0, T), H^{1}(0,1)\right)$. In order to show that this limit is the solution of problems (77)-(79), it is sufficient to demonstrate that $h$ verifies (64) and (72).

We have, from problems (74)-(76), that

$$
R\left(h^{(n)}, u\right)=\left(u, \Im_{x} G\left(x, t, h^{(n-1)}, \frac{\partial h^{(n-1)}}{\partial x}\right)\right)_{L^{2}(D)}
$$

Precisely

$$
\begin{aligned}
R\left(h^{(n)}-h, u\right)+R(h, u)= & \left(u, \Im_{x} G\left(x, t, h^{(n-1)}, \frac{\partial h^{(n-1)}}{\partial x}\right)-\Im_{x} G\left(x, t, h, \frac{\partial h}{\partial x}\right)\right)_{L^{2}(D)} \\
& +\left(u, \Im_{x} G\left(x, t, h, \frac{\partial h}{\partial x}\right)\right)_{L^{2}(D)}
\end{aligned}
$$

using Equation (74), then (95) becomes

$$
\begin{aligned}
R\left(h^{(n)}-h, u\right)= & -\left({ }^{c} \partial_{t}^{\beta+1} \mathfrak{\Im}_{x}\left(h^{(n)}-h\right), u\right)_{L^{2}(D)}+\left(\gamma \frac{\partial\left(h^{(n)}-h\right)}{\partial x}, u\right)_{L^{2}(D)} \\
& +\left(\frac{\partial}{\partial t}\left(\eta \frac{\partial\left(h^{(n)}-h\right)}{\partial x}\right), u\right)_{L^{2}(D)}+\left(\int_{0}^{t} \xi(t-z) \mathfrak{\Im}_{x}\left(h^{(n)}-h\right)(x, z) d z, u\right)_{L^{2}(D)}
\end{aligned}
$$


By integrating the parts on all terms on the LHS, and taking into consideration conditions on $v$ and $w$, (96) transforms into

$$
\begin{aligned}
R\left(h^{(n)}-h, u\right)= & -\left({ }^{C} \partial_{t}^{\beta+1}\left(h^{(n)}-h\right), \Im_{x} u\right)_{L^{2}(D)}+\left(\gamma \frac{\partial\left(h^{(n)}-h\right)}{\partial x}, u\right)_{L^{2}(D)} \\
& +\left(\eta \frac{\partial\left(h^{(n)}-h\right)}{\partial x}, \frac{\partial u}{\partial t}\right)_{L^{2}(D)}+\left(\int_{0}^{t} \xi(t-z) \Im_{x}\left(h^{(n)}-h\right)(x, z) d z, u\right)_{L^{2}(D)}
\end{aligned}
$$

Applying Cauchy-Schwartz inequality yields

where

$$
R\left(h^{(n)}-h, u\right) \leq \delta_{13}\left\|h^{(n)}-h\right\|_{L^{2}\left(0, T, H^{1}(\Omega)\right)}\left(\|u\|_{L^{2}((D)}+\left\|\frac{\partial u}{\partial t}\right\|_{L^{2}((D)}\right)
$$

and from (95) we have the following estimate

$$
\delta_{13}=\max \left(c_{1}+T \frac{c_{0}}{2}, c_{6}\right)
$$

$$
\begin{aligned}
& \left(u, \Im_{x} G\left(x, t, h^{(n-1)}, \frac{\partial h^{(n-1)}}{\partial x}\right)-\Im_{x} G\left(x, t, h, \frac{\partial h}{\partial x}\right)\right)_{L^{2}\left(D^{T}\right)} \leq \frac{L}{\sqrt{2}}\left\|h^{(n)}-h\right\|_{L^{2}\left(\left(0, T, H^{1}(\Omega)\right)\right.} \\
& \|u\|_{L^{2}(D)}
\end{aligned}
$$

Passing to the limit $n \rightarrow \infty$ in (97), and taking into consideration (98)-(99), we obtain

$$
R(h, u)=\left(u, \Im_{x} G\left(x, t, h, \frac{\partial h}{\partial x}\right)\right)_{L^{2}(D)}
$$

To conclude that problems (77)-(79) admit a weak solution, we prove that (64) holds. Since $\lim _{n \rightarrow \infty}\left\|h^{(n)}-h\right\|_{L^{2}\left(\left(0, T ; H^{1}(\Omega)\right)\right.}=0$ then, we deduce that $\int_{0}^{1} h d x=0$ and $\frac{\partial h}{\partial x}(1, t)=0$.

Therefore, we have established this result:

Theorem 12. Suppose that conditions of Lemma (11) hold, and that $L<\sqrt{\frac{\min (1, T)}{\delta_{11} T}}$, then the problems (62)-(64) admit a weak solution in $L^{2}\left(0, T, H^{1}(\Omega)\right)$.

Now, we prove the uniqueness of problems (62)-(64).

Theorem 13. Under conditions of Lemma (11), the problems (62)-(64) admits unique solutions.

Proof. Suppose that the problems (62)- (64) admit $v_{1}$ and $v_{2}$ as solutions in $L^{2}\left(0, T, H^{1}(\Omega)\right)$, then $H=v_{1}-v_{2}$ belongs to $L^{2}\left(0, T, H^{1}(\Omega)\right)$ and verifies

$$
\begin{gathered}
\mathcal{L} H={ }^{C} \partial_{t}^{\beta+1} H-\frac{\partial}{\partial x}\left(\gamma(x, t) \frac{\partial H}{\partial x}\right)-\frac{\partial^{2}}{\partial x \partial t}\left(\eta(x, t) \frac{\partial H}{\partial x}\right)-\int_{0}^{t} \xi(t-z) H(x, z) d z=\Psi(x, t) \\
\ell_{1} H=H(x, 0)=0, \quad \ell_{2} H=H_{t}(x, 0)=0, \quad x \in \Omega, \\
\int_{0}^{1} H(x, t) d x=0, \quad H_{x}(1, t)=0, \quad t \in(0, T),
\end{gathered}
$$

where $\Psi(x, t)=G\left(x, t, v_{1}, \frac{\partial v_{1}}{\partial x}\right)-G\left(x, t, v_{2}, \frac{\partial v_{2}}{\partial x}\right)$. 
This will be done by establishing the same proof of Lemma 11; we obtain

$$
\|H\|_{L^{2}\left(0, T, H^{1}(\Omega)\right)} \leq K\|H\|_{L^{2}\left(0, T, H^{1}(\Omega)\right)}
$$

Since $K<1$, then from (80) we have $(1-K)\|H\|_{L^{2}\left(0, T, H^{1}(\Omega)\right)} \leq 0$, from which we deduce that $v_{1}=v_{2}$ in $L^{2}\left((0, T), H^{1}(\Omega)\right)$.

\section{References}

1. Mesloub S, Aldosari F, Even higher order fractional initial boundary value problem with nonlocal constraints of purely integral type. Symmetry. 2019; 11(3): 305.

2. Mesloub S, Algahtani O, On a singular nonlocal time fractional order mixed problem with a memory term. Math Methods Appl Sci. 2018; 41(12): 4676-90.

3. Mesloub S, Existence and uniqueness results for a fractional two-times evolution problem with constraints of purely integral type. Math Methods Appl Sci. 2016; 39(6): 1558-67.

4. Mesloub S, A nonlinear nonlocal mixed problem for a second order pseudoparabolic equation. J Math Anal Appl. 2006; 316(1): 189-209.

5. Bouziani A, Solvability of nonlinear pseudoparabolic equation with a nonlocal boundary condition, Nonlinear Anal Theor Methods Appl. 2003; 55(7-8): 883-904.

6. Elbeleze AA, Kilicman A, Taib BM, Application of homotopy perturbation and variational iteration methods for fredholm integrodifferential equation of fractional order, Abstract and applied analysis: Hindawi, 2012; 2012.

7. Sofiane D, Abdelfatah B, Taki-Eddine O, Study of solution for a parabolic integrodifferential equation with the second kind integral condition. Int J Anal App. 2018; 16(4): 569-93.

8. Cassani D, Vilasi L, Wang Y, Local versus nonlocal elliptic equations: short-long range field interactions. Adv Nonlinear Anal. 2021; 10(1): 895-921.

9. Correa E, de Pablo A, Remarks on a nonlinear nonlocal operator in orlicz spaces. Adv Nonlinear Anal. 2020; 9(1): 305-26. doi: 10.1515/anona-2020-0002.

10. Balachandran K, Uchiyama $\mathrm{K}$, et al. Existence of solutions of quasilinear integrodifferential equations with nonlocal condition. Tokyo J Math. 2000; 23(1): 203-10.

11. Merad A, Martín-Vaquero J, A galerkin method for two-dimensional hyperbolic integrodifferential equation with purely integral conditions. Appl Math Comput. 2016; 291: 386-94.

12. Chergui D, Oussaeif TE, Ahcene M, Existence and uniqueness of solutions for nonlinear fractional differential equations depending on lower-order derivative with non-separated type integral boundary conditions. AIMS Math. 2019; 4(1): 112.

13. Merad A, Bouziani A, Cenap O, Kilicman A, On solvability of the integrodifferential hyperbolic equation with purely nonlocal conditions. Acta Math Sci. 2015; 35(3): 601-9.

14. Mingqi X, Rădulescu VD, Zhang B, Nonlocal Kirchhoff diffusion problems: local existence and blow-up of solutions. Nonlinearity. 2018; 31(7): 3228.

15. Tang X, Chen S, Ground state solutions of nehari-pohozaev type for Kirchhoff-type problems with general potentials. Calc Var Partial Differ Equ. 2017; 56(4): 1-25.

16. Daftardar-Gejji V, Jafari H, Adomian decomposition: a tool for solving a system of fractional differential equations. J Math Anal Appl. 2005; 301(2): 508-18.

17. Merad A, Adomian decomposition method for solution of parabolic equation to nonlocal conditions. Int J Contemp Math Sci. 2011; 6(30): 1491-96.

18. Merad A, Bouziani A, Cenap O, Inversion laplace transform for integrodifferential parabolic equation with purely nonlocal conditions. Hacet J Math Stat. 2015; 44(5): 1087-97.

19. Aghili A, Fractional black-scholes equation. Int J Financ Eng. 2017; 4(1): 1750004. 
20. Pandir Y, Duzgun HH, New exact solutions of time fractional gardner equation by using new version of f-expansion method. Commun Theor Phys. 2017; 67(1): 9.

21. Tang XH, Cheng B, Ground state sign-changing solutions for Kirchhoff type problems in bounded domains, J Diff Eq. 2016; 261(4): 2384-402.

22. Fardi M, Ghasemi M, Solving nonlocal initial-boundary value problems for parabolic and hyperbolic integro-differential equations in reproducing kernel hilbert space. Numer Methods Partial Diff Eq. 2017; 33(1): 174-98.

23. Niu J, Lin Y, Cui M, Approximate solutions to three-point boundary value problems with twospace integral condition for parabolic equations. Abstract and applied analysis: Hindawi. 2012; 2012.

24. Taki-Eddine O, Abdelfatah B, A priori estimates for weak solution for a time-fractional nonlinear reaction-diffusion equations with an integral condition, Chaos. Sol Frac. 2017; 103: 79-89.

25. Wang Y, Du M, Tan F, Li Z, Nie T, Using reproducing kernel for solving a class of fractional partial differential equation with non-classical conditions. Appl Math Comput. 2013; 219(11): 5918-25.

26. Mesloub S, Bachar I, On a singular time-fractional order wave equation with bessel operator and caputo derivative. J Nonlinear Sci Appli. 2017; 10(1): 60-70.

27. Belmouloud I, Memou A, On the solvability of a class of nonlinear singular parabolic equation with integral boundary condition. Appl Math Comput. 2020; 373: 124999.

28. Oussaeif TE, Bouziani A, Solvability of nonlinear goursat type problem for hyperbolic equation with integral condition. Khayyam J Math. 2018; 4(2): 198-213.

29. Alikhanov A, A priori estimates for solutions of boundary value problems for fractional-order equations. Differ Equ. 2010; 46(5): 660-6.

30. Mesloub S, Mezhoudi R, Medjeden M, 8. a mixed problem for a parabolic equation of higher order with integral conditions. Bull Polish Acad Sci Math. 2002; 50(3): 313-22.

31. Ladyzhenskaya OA, The boundary value problems of mathematical physics. Springer Sci Bus Media. 2013; 49: 322.

32. Mesloub S, Bouziani A, On a class of singular hyperbolic equation with a weighted integral condition. Int J Math Math Sci. 1999; 22: 511-19.

\section{Corresponding author}

Sakhri Aicha can be contacted at: sakhri.aicha@univ-oeb.dz

For instructions on how to order reprints of this article, please visit our website:

www.emeraldgrouppublishing.com/licensing/reprints.htm

Or contact us for further details: permissions@emeraldinsight.com 